\title{
The effect of the nonlinear viscoelastic properties on tack
}

\author{
C. Verdier, J.-M. Piau
}

Laboratoire de Rhéologie

UJF-INPG, CNRS (UMR 5520)

BP 53 - Domaine Universitaire

38041 Grenoble Cedex 9

\begin{abstract}
:
The influence of rheological and surface properties on adhesive tackiness are studied. In particular, the importance of the elongational properties is emphasized in a model, which considers only the adhesive contribution, while neglecting the importance of cavitation and surface roughness. It is shown that this simple analysis allows one to recover the different types of curves (i.e. different adhesive materials) obtained in the literature on tack. Elastic, strain hardening and viscous adhesive materials are considered. The question of the importance of surface properties is raised and discussed.
\end{abstract}

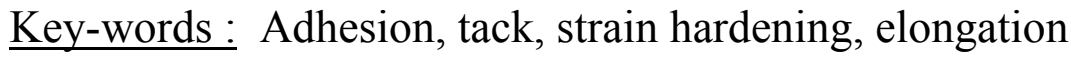




\section{Introduction}

Testing adhesive materials is not an easy task and requires very careful experiments with adequate surface properties (type, roughness) and surface treatments (chemical, plasma, etc.) as well as optimally designed mechanical equipments. In addition, the industrial adhesive material is sometimes not so well defined : various chemical components are used, and their migration is possible, thus changing the interfacial properties. Tack is one of the widely used tests [1-3], which provides good data. Usually, the contact time is quite fast (a few seconds), which is interesting since this limits diffusion, as well as surface pollution. On the other hand, wetting is limited, because it is a long process for such materials (high viscosities or moduli). As a consequence, air (or water) may be present in the surface holes and will lead to cavitation [1-2], as the probe moves away. Cavitation is important, for it can sometimes control the adhesive deformations: small cavities at the interface appear and grow until they eventually meet, while fibrils are elongated in between. Two kinds of hole sizes may be present [4] due to roughness of both adhesive and probe. The differences in roughness peaks can create significant cavities and seem to affect adhesion also [5], as well as viscoelastic effects [6]. At present, no experiments on a perfectly flat surface treated properly, using a perfect probe have yet been reported, because of the difficulties inherent to such systems. This constitutes a real challenge.

Deformations involved during tack are not obvious to understand. During the first instants, shear is dominant, as well as cavitation. Then elongation is important because of the formation of fibrils at large times [1]. This complicates the problem further.

The engineering stress is usually reported as a function of the engineering strain in such experiments, which is convenient. Such plots show different regimes, which can be obtained by varying either the temperature, the contact time, the velocity of the probe, or the cross-linking density [1]. Such plots have not been explored completely, and it is the purpose of this work to try to present a simplified model taking into account 
the importance of the rheological properties, through the use of various possible adhesives (Newtonian, viscoelastic, elastic) to explain these features. The starting idea is therefore one already proposed by the authors, when studying peeling tests, related to the fact that the elongation properties of the adhesive are important [7-9]. But it has also been foreseen in the famous picture from Kaelble's paper [10]. He actually describes a simple peeling experiment where fibrils born from cavities are elongated, when moving towards the peeling front. Such correspondence between tack and peel is not a coincidence: it reveals that the mechanisms present in both experiments are strongly correlated, as mentioned before [11]. Based on these observations, we will construct a model that contains the basic assumption, i.e. the elongation of the adhesive material.

In the first part $(\S 2)$ of this work, tack will be described, followed by the analysis of the type of deformation that the adhesive undergoes. Then the adhesive material will be presented in the next section ( $\$ 3)$, with a simple heuristic model accounting for most adhesive behaviors. In the next section (§4), the force-deformation curve will be obtained. This will be followed by presentation of such data (§5), using a rheological model. Finally, results will be discussed in the last part (§6).

\section{Description of tack}

\subsection{Experiment}

Tack has been studied experimentally quite intensively [1-3,12-13] and is a simple test where a cylindrical probe is brought into contact with a thin film of adhesive (thickness e, typically 50-300 $\mu \mathrm{m}$ ) for a short time (a few seconds generally). Then the probe is pulled away at constant velocity V (Figure 1). V usually ranges between 0.001 $\mu \mathrm{m} / \mathrm{s}$ and $100 \mu \mathrm{m} / \mathrm{s}$. Although tack is a rather simple test, it is important to list here the different parameters, which may play a role in such an experiment:

- adhesive rheometrical or mechanical properties, degree of cross-linking

- adhesive thickness (e) and cross-section $\left(\mathrm{A}_{0}\right)$

- adhesive radius size compared to probe size.

- velocity of the probe $(\mathrm{V})$ and force $F(t)$ 
- contact time $\left(\mathrm{t}_{\mathrm{c}}\right)$ and application pressure $(\mathrm{P})$

- temperature $(\mathrm{T})$ and relative humidity $(\mathrm{RH})$

- surface treatment, surface roughness (substrate and probe)
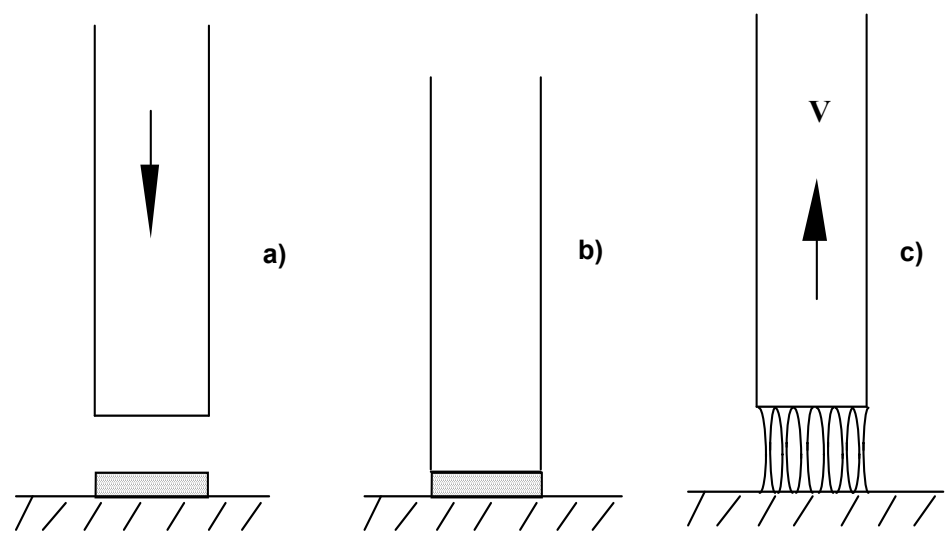

\section{Figure 1. Sketch of a tack experiment (no edges)} a) approach b) contact c) separation at constant velocity $\mathrm{V}$

These parameters should all be taken into account and are lacking in relevant papers. In particular, changing the dimensions of the adhesive can change the boundary conditions - and therefore the solution - of the corresponding problem. As mentioned before, the engineering stress $\sigma_{\mathrm{e}}(\mathrm{t})=\mathrm{F}(\mathrm{t}) / \mathrm{A}_{0}$ is commonly used to represent the tackiness of the material. Instead of representing it as a function of time, one can define the engineering strain $\varepsilon_{\mathrm{e}}=\mathrm{Vt} / \mathrm{e}$, where $\mathrm{t}$ is time, and $\mathrm{V}$ and $\mathrm{e}$ have been defined previously.

Curves showing force vs. time can be found in the literature [1-3] and are summarized in Figure 2. When pulling on a viscous adhesive (2a), the force goes up almost instantaneously then decreases slowly to zero, while a long filament is formed. For a viscoelastic material (2b), the stress increases sharply, goes through a maximum, and decreases again, then possibly shows a plateau before dropping to zero. Fibrillation initiates after the peak load and the deformation of the fibrils occurs in the plateau region [1-2]. The plateau may sometimes be followed by a slight increase in force, this being attributed to strain hardening within the fibrils. On the other hand, for an elastic 
adhesive (2c), the stress increases sharply until the adhesive fails rapidly. Lowering the temperature, increasing the velocity, increasing the contact time, or again increasing the cross-linking density of the polymer [1] all correspond to going from (a) to (b), then (c). In fact, this is not surprising when one recalls the time-temperature superposition principle, which accounts for such correlations.

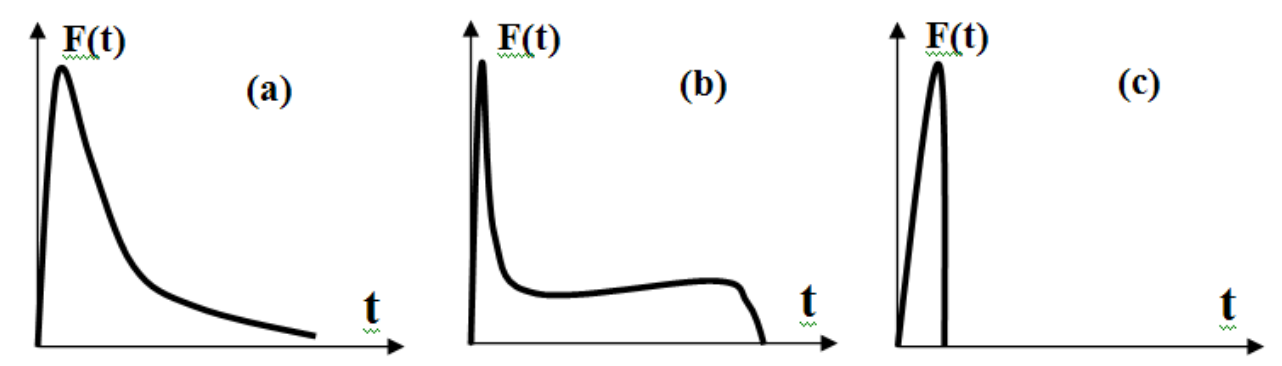

Figure 2. Different types of tack curves (a) Newtonian (b) viscoelastic (c) elastic

Another problem is to understand whether rupture is at the interface (adhesive) or within the material (cohesive). Usually, for most Newtonian adhesives, failure is within the sample, after a long filament has been formed. For an elastic adhesive, failure is frequently located at the substrate-adhesive interface. This has been shown for example in the case of polymer melts [12]. In the intermediate cases (so-called viscoelastic systems), it is not so clear whether failure is in the bulk or at the interface. A nice answer to this question has been provided [13], providing a classification into different modes of failure: edge crack propagation, internal crack propagation, cavitation and bulk fingering. Since cavitation may occur, it is very helpful to observe this process from a side view and also from below [2], in order to understand how cavities grow [14] and how they affect the adhesive deformations. Such observations may also allow to see where crack propagation occurs, either at the adhesive-substrate or adhesive-probe interfaces. In such cases, local peeling is possible. On the other hand, the material may fail cohesively. A failure criterion can be introduced [15] through a 
dimensionless number $\Lambda$, which represents the effects of the elongation stresses compared to the ones due to surface energies. If $\Lambda$ is greater than 1 (respectively $\Lambda$ smaller than 1), then interfacial failure occurs (respectively bulk failure happens). This criterion has unfortunately not been used further, although it could help to understand principles such as the energy separation criterion [16] and its range of application.

Understanding the shapes of the curves in Figure 2 is not so simple, due to the different mechanisms encountered: cavitation, fibrillation, etc. Actually no model exists, which accounts for the overall shape of such plots. Cavitation has been studied experimentally [2] and theoretically [4,17], but apparently no model is yet available due the complexity of the cavitation process, except in the particular case of the elastic adhesive [4]. Another limiting parameter is surface roughness [5], which interplays with viscoelasticity [6], and renders modeling more difficult. It is now clear that shear and cavitation are present at the beginning of the test, whereas fibrillation is important at the end [1-2]. This is what is considered next.

\subsection{Shear vs. elongation : the Newtonian case}

Let us start with a general picture of a probe pulling a Newtonian adhesive. This ideal case will be helpful to proceed further. In this case, the problem can be dealt with using general conservation laws. Figures 3a-b show a representation of the deformed adhesive, at the beginning when shear starts towards the edge (Figure 3a), and later on (Figure $3 b$ ) as elongation becomes important. Figure $3 b$ correspond to a Newtonian adhesive and the case of a viscoelastic one is more likely to be portrayed as in Figure 1c. Understanding the different processes (shear or elongation or both) can be done by comparing two forces, the one required to create a shear flow within the adhesive (initial times) and the one necessary to elongate the adhesive.

Assuming the radius $\mathrm{R}(\mathrm{t})$ at the mid-section is almost a constant, a mass balance gives:

$$
\pi \mathrm{R}(\mathrm{t})^{2} \mathrm{~h}(\mathrm{t})=\pi \mathrm{R}_{0}^{2} \mathrm{e}
$$


where $\mathrm{R}_{0}$ is the initial adhesive radius (usually around $10 \mathrm{~mm}$ ), and $\mathrm{h}(\mathrm{t})$ is the actual adhesive length.

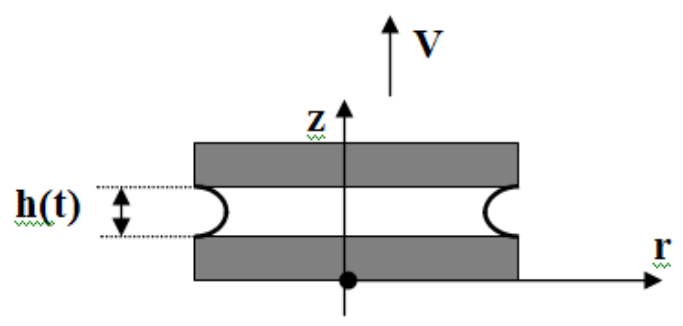

a)

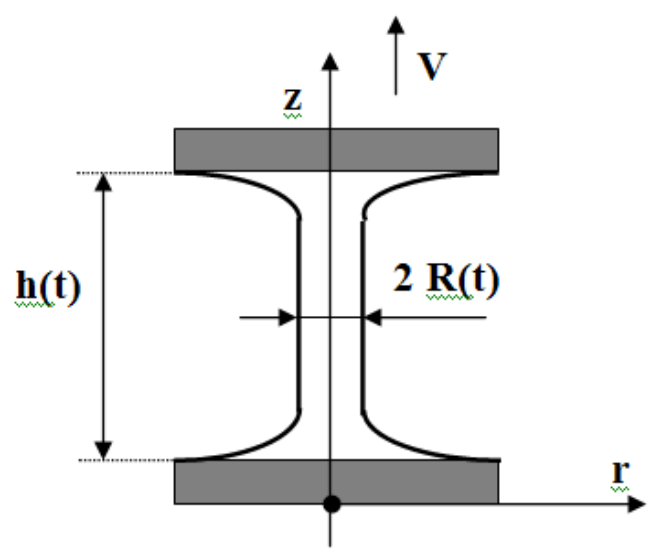

b)

Figure 3. Shape of the adhesive (initial thickness $=$ e). Probe is pulled at constant velocity V. a) Short times: shear dominates

b) long times : elongation prevails

It is to be understood here that the ratio $\mathrm{e} / \mathrm{R}_{0}$ is small (of the order 0.01 ) and that shear flow (starting at the edge, Figure 3a) has to take place at the first instants in order for the adhesive to deform, because of the large hydrostatic tension. At this point, the adhesive may cavitate, but this will not be taken into account because we consider a thoroughly degassed fluid. Let us now determine typical times corresponding to the cases of the shear flow and the uniaxial elongational flow .

In the case of a shear flow, the lubrication approximation may be used to obtain the simplified momentum equation:

$$
\frac{\partial \mathrm{p}}{\partial \mathrm{r}}=\eta_{0} \frac{\partial^{2} \mathrm{u}_{\mathrm{r}}}{\partial \mathrm{z}^{2}}
$$


$\mathrm{p}(\mathrm{r})$ is the pressure, $\eta_{0}$ is the zero-shear viscosity, $\mathrm{u}_{\mathrm{r}}(\mathrm{z})$ is the radial velocity component, $\mathrm{r}$ the radial distance, and $\mathrm{z}$ the vertical distance. The boundary conditions are: $\mathrm{u}_{\mathrm{r}}(\mathrm{z}=0)=0, \mathrm{u}_{\mathrm{r}}(\mathrm{z}=\mathrm{h})=0$ and $\mathrm{p}(\mathrm{r}=\mathrm{R})=0$. The velocity $\mathrm{u}_{\mathrm{r}}$ is obtained by integration from 0 to $\mathrm{h}$ by standard calculation, and its average is:

$$
\overline{\mathrm{u}}_{\mathrm{r}}=\frac{-\mathrm{h}^{2}}{12 \eta_{0}} \frac{\partial \mathrm{p}}{\partial \mathrm{r}}
$$

Now from mass conservation, we have $\overline{\mathrm{u}_{\mathrm{r}}} \approx \frac{-\mathrm{r}}{2 \mathrm{~h}} \dot{\mathrm{h}}$, therefore

$$
\frac{\partial \mathrm{p}}{\partial \mathrm{r}}=6 \eta_{0} \frac{\dot{\mathrm{h}}}{\mathrm{h}^{3}} \mathrm{r}
$$

Finally, we integrate from $\mathrm{R}(\mathrm{t})$ to $\mathrm{r}$ to obtain the pressure:

$$
\mathrm{p}(\mathrm{r})=3 \eta_{0} \frac{\dot{\mathrm{h}}}{\mathrm{h}^{3}}\left(\mathrm{r}^{2}-\frac{\mathrm{e}}{\mathrm{h}} \mathrm{R}_{0}^{2}\right)
$$

where $\mathrm{R}$ has been eliminated using mass conservation.

The force $F_{S}(t)$ corresponding to such a shear flow is then given by integration of the pressure along the surface $F_{S}(t)=\int^{R} p(r) 2 \pi r$ dr. This leads to:

$$
\mathrm{F}_{\mathrm{S}}(\mathrm{t})=\frac{3 \eta_{0}}{2} \frac{\pi \mathrm{R}_{0} 4}{\mathrm{e}^{2}} \frac{\mathrm{V}}{\mathrm{e}} \frac{1}{(1+\mathrm{Vt} / \mathrm{e})^{5}}(6)
$$

This corresponds to the force required to shear the Newtonian adhesive with viscosity $\eta_{0}$ when no cavitation occurs.

In the case of pure elongation, the calculation is standard. The force $\mathrm{F}_{\mathrm{e}}(\mathrm{t})$ is given by :

$$
\mathrm{F}_{\mathrm{e}}(\mathrm{t})=3 \eta_{0} \dot{\varepsilon}(\mathrm{t}) \pi \mathrm{R}(\mathrm{t})^{2}
$$

where $\dot{\varepsilon}$ is the time-dependent strain rate and is given once the Hencky strain $\varepsilon(\mathrm{t})$ is known:

$$
\varepsilon(t)=\operatorname{Ln}\left(\frac{e+V t}{e}\right)
$$

Therefore, the strain rate is:

$$
\dot{\varepsilon}(\mathrm{t})=\frac{\mathrm{V}}{\mathrm{e}+\mathrm{Vt}}
$$

The strain rate decreases to zero as a function of time. We note that for small times it goes like V/e (a constant) while it varies as $1 / \mathrm{t}$ for large times. This point will 
be used later. Another important feature is the correspondence between the real strain (Hencky) and the engineering strain, which is no longer valid at large times. Indeed the engineering strain Vt/e would simply predict a constant strain rate (V/e) experiment, which is not the case. Usually, conventional rheometrical tests use a constant strain rate, corresponding to an exponential stretch of the filament. Going back to the force, using (1), (7) and (9), we obtain:

$$
F_{e}(t)=3 \eta_{0} \pi R_{0}^{2} \frac{V}{e} \frac{1}{(1+V t / e)^{2}}
$$

To determine what type of flow prevails, we need to compare $F_{S}(t)$ and $F_{e}(t)$ from equations (6) and (10):

$$
\frac{\mathrm{F}_{\mathrm{S}}(\mathrm{t})}{\mathrm{F}_{\mathrm{e}}(\mathrm{t})}=\frac{\mathrm{R}_{0}{ }^{2}}{2 \mathrm{e}^{2}(1+\mathrm{Vt} / \mathrm{e})^{3}}
$$

We conclude that the shear force dominates at small times ( ince $\mathrm{R}_{0} / \mathrm{e} \approx 100$ ), as expected. Then for a typical time, the elongational regime appears i.e. when $t>\frac{\mathrm{e}}{\mathrm{V}}$, so that

$$
\frac{\mathrm{F}_{\mathrm{S}}(\mathrm{t})}{\mathrm{F}_{\mathrm{e}}(\mathrm{t})} \approx \frac{\mathrm{R}_{0}{ }^{2}}{2 \mathrm{e}^{2}(\mathrm{Vt} / \mathrm{e})^{3}}
$$

This term becomes of the order 1 when $\mathrm{t} \approx \frac{\mathrm{e}}{\mathrm{V}}\left(\frac{\mathrm{R}_{0}}{\mathrm{e}}\right)^{2 / 3}$.

Finally three regimes are obtained:
a) $\mathrm{t}<\frac{\mathrm{e}}{\mathrm{V}}$
Shear dominates
b) $\frac{\mathrm{e}}{\mathrm{V}}<\mathrm{t}<\frac{\mathrm{e}}{\mathrm{V}}\left(\frac{\mathrm{R}_{0}}{\mathrm{e}}\right)^{2 / 3}$
Mixed shear and elongation
c) $\mathrm{t}>\frac{\mathrm{e}}{\mathrm{V}}\left(\frac{\mathrm{R}_{0}}{\mathrm{e}}\right)^{2 / 3}$
Elongation prevails

The last important time to add will be the characteristic time of the material, when the latter becomes viscoelastic, and this will be considered next.

\section{The adhesive material}


Considering now a real adhesive, the Newtonian case should be a limit of the viscoelastic material at large times, and the elastic behavior should correspond to very rapid solicitations. We will assume that the adhesive is characterized by one relaxation time $\lambda$ although it is not hard to generalize our model to multiple relaxation times. Details of dynamic data for a usual adhesive can be found elsewhere [8] and will not be discussed. The elongational viscosity is important since our model will be based on the assumption that most of the deformations are of elongational type, except at short times. Usually, rheometrical measurements use constant elongation rate $\dot{\varepsilon}$. As seen from (9), this is not the case anymore. Therefore, we need to exhibit a basic traction curve where the kinematics correspond to a tack experiment, i.e. at constant tack velocity V. This type of test has occasionally been carried out [18]. In Figure 4, we show the evolution of a typical transient elongational viscosity $\eta^{+}(\mathrm{t})$ against time, in a constant velocity experiment (Fig 4a). Usually, the viscosity $\eta^{+}(t)$ increases with a slope of 1 at short times (log-log scale), followed by a possible plateau, and then a rapid exponential increase corresponds to strain hardening. The characteristic time $\lambda$ is also shown. Three typical parts can be identified:

$$
\begin{array}{llrl}
\eta^{+}(\mathrm{t}) & =\mathrm{K}_{0} \mathrm{t} & 0<\mathrm{t}<\lambda \\
\eta^{+}(\mathrm{t}) & =3 \eta_{0}(1-\exp (-\mathrm{t} / \lambda)) & & \lambda<\mathrm{t}<\mathrm{t}_{1} \\
\eta^{+}(\mathrm{t}) & =3 \eta_{0} \exp \left(\mathrm{K}\left(\mathrm{t}-\mathrm{t}_{1}\right)\right) & & \mathrm{t}>\mathrm{t}_{1}
\end{array}
$$

$\mathrm{K}_{0}$ is in fact equal to $3 \eta_{0} / \lambda$, as a limiting case of $(12)_{\mathrm{b}}$ for short times and is a material parameter, whereas $\mathrm{K}$ and $\mathrm{t}_{1}$ may be velocity $(\mathrm{V})$ dependent. Formulae $(12)_{\mathrm{b}-\mathrm{c}}$ are for the viscoelastic adhesive respectively at small and high stretch rates V/e.

Then we plot the true elongation rate vs. time in logarithmic coordinates (Fig4b). As discussed before, it is a constant V/e at small times, then it decreases and shows a slope -1 at large times $(1 / \mathrm{t})$. The time $\mathrm{e} / \mathrm{V}$ is also represented along the time axis. Then the evolution of the first normal stress difference $\sigma=\sigma_{\mathrm{zz}}-\sigma_{\mathrm{rr}}=\eta^{+}(\mathrm{t}) \dot{\varepsilon}(\mathrm{t})$ is shown in Figure 4c. $\sigma_{\mathrm{zz}}$ and $\sigma_{\mathrm{rr}}$ are the principal stresses in the uniaxial test. $\sigma$ is required here because the force transducer measures it; also $\sigma_{\mathrm{zz}}-\sigma_{\mathrm{rr}}$ is commonly used in rheometrical measurements. This stress difference $\sigma$ increases first and goes through a maximum, 
then it decreases until strain hardening occurs and we obtain a very rapid increase (exponential). This exponential increase will be even more substantial as the velocity $\mathrm{V}$ increases. Finally, we plot $\sigma$ as a function of the Hencky strain $\varepsilon=\operatorname{Ln}(1+\mathrm{Vt} / \mathrm{e})$; the picture is similar (Fig 4d), although the data is squeezed at short times.

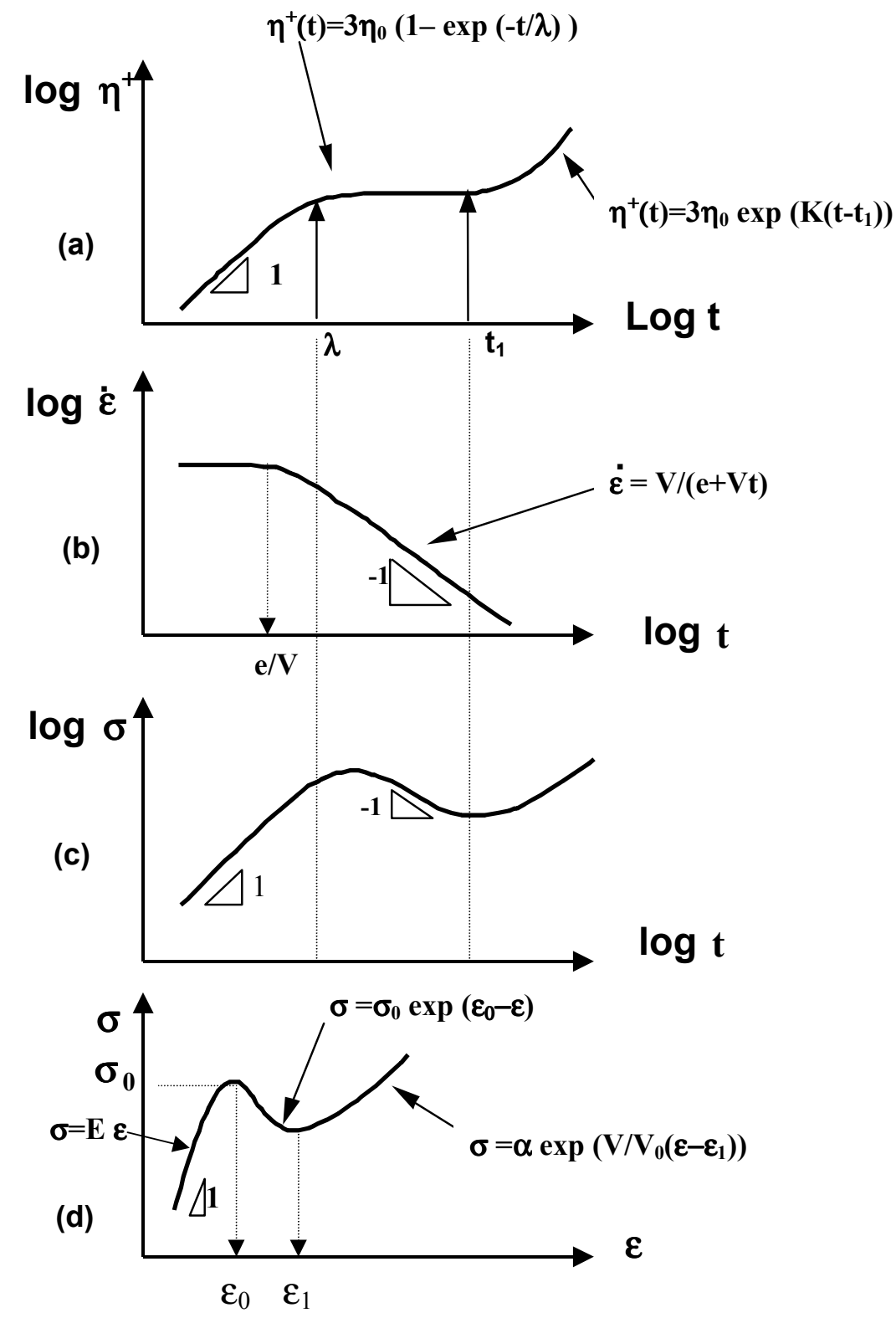

Figure 4. Typical plots showing (a) transient elongational viscosity (b) strain rate (c) transient stress difference (d) stress difference-strain curve

The case in Figure 4 represents a viscoelastic adhesive. Parts of the branches correspond to elastic and Newtonian behavior. They can be obtained depending on 
$\lambda$ and $\mathrm{e} / \mathrm{V}$. Indeed, when $\lambda<<\mathrm{e} / \mathrm{V}$, a Newtonian behavior is obtained. On the contrary, when $\lambda \gg>\mathrm{e} / \mathrm{V}$, an elastic response is found. The intermediate case corresponding to $\lambda \approx \mathrm{e} / \mathrm{V}$ (Fig 4 , for example, $\lambda$ slightly higher than $\mathrm{e} / \mathrm{V}$ ) is the case of major interest, because it corresponds to the viscoelastic adhesive.

Equations corresponding to the different branches are also given in Figs 4a-d. The following stress-strain laws can represent the adhesive material:

* Newtonian adhesive: $\quad \varepsilon_{0} \rightarrow 0$ and $\varepsilon_{1 \rightarrow \infty}$

$$
\sigma(\varepsilon)=\sigma_{0} \exp (-\varepsilon)
$$

* Viscoelastic strain hardening adhesive: (parameters $\varepsilon_{0}$ and $\varepsilon_{1}, \mathrm{E}, \mathrm{V}_{0}$ )

$$
\begin{array}{ll}
\varepsilon<\varepsilon_{0} & \sigma(\varepsilon)=\mathrm{E} \varepsilon \\
\varepsilon_{0}<\varepsilon<\varepsilon_{1} & \left.\sigma(\varepsilon)=\sigma_{0} \exp \left(\varepsilon_{0}-\varepsilon\right) \quad \quad \text { (where } \sigma_{0}=\mathrm{E} \varepsilon_{0}\right) \\
\varepsilon>\varepsilon_{1} & \sigma(\varepsilon)=\sigma_{0} \exp \left(\varepsilon_{0}-\varepsilon_{1}\right) \exp \left\{\mathrm{V} / \mathrm{V}_{0}\left(\varepsilon-\varepsilon_{1}\right)\right\}
\end{array}
$$

* Elastic adhesive: $\left(\varepsilon_{0} \rightarrow \infty\right.$ and $\left.\varepsilon_{1} \rightarrow \infty\right)$

$$
\sigma(\varepsilon)=\mathrm{E} \varepsilon \quad \text { small deformations }
$$

The material parameters are enough to capture all the characteristics of the adhesive. There are four independent parameters (strains $\varepsilon_{0}, \varepsilon_{1}$, E the elastic modulus, and $\mathrm{V}_{0}$, a characteristic velocity) and by adjusting them we find the limiting cases mentioned previously, i.e. Newtonian and elastic adhesives. These expressions are important because they can be easily integrated (see $\S 6$ ).

To determine such parameters for a real adhesive, we suggest to evaluate both the shear and elongational properties and to use a correct constitutive equation, like Lodge's model, which will be presented in $§ 5$.

The following analysis will be based on this model, and an attempt to recover the three typical curves in a tack experiment will be made. The analysis can be done in terms of time t or Hencky strain $\varepsilon$.

\section{Model}


The main idea is to look at the importance of the elongational regime for describing tack. Therefore, we limit the study to the following assumptions:

- homogeneous elongational deformation

- constant velocity

- perfectly flat surfaces (no roughness)

- perfect preparation : no pollution, no air entrapped

- cavitation effects neglected

- force additivity in fibrils

The last point needs to be explained in detail. We assume that at a given time t, the force is $\sigma(\mathrm{t})$ in a filament and the global force is $\mathrm{F}(\mathrm{t})$. By force additivity, we mean that if the fibril is split into two different ones (or more) due to cavitation, the stress $\sigma(t)$ remains unchanged in each fibril and the force is the sum $\sigma(\mathrm{t}) \mathrm{A}(\mathrm{t}) / 2+\sigma(\mathrm{t}) \mathrm{A}(\mathrm{t}) / 2=\sigma(\mathrm{t})$ $A(t)$. In other words the force is unchanged. As a consequence, the surface area to be considered is the total cross-section of all the filaments. The latter one is found using mass conservation:

$$
\mathrm{A}(\mathrm{t})(\mathrm{e}+\mathrm{Vt})=\mathrm{A}_{0} \mathrm{e}
$$

Finally the force to elongate the adhesive, or the set of fibrils, will simply be given by:

$$
F(t)=\sigma(t) A(t)=\sigma(t) A_{0} \frac{e}{e+V t}
$$

Using the Hencky strain instead, the force can also be given by :

$$
\mathrm{F}(\varepsilon)=\sigma(\varepsilon) \mathrm{A}_{0} \exp (-\varepsilon)
$$

where $\sigma(\varepsilon)$ is given by Eqs (13)-(14)-(15). To complete the model, we need to add a final criterion for $\varepsilon$, say $\varepsilon_{\mathrm{f}}$ (velocity dependent one), but this will be discussed in the next section. This criterion is different in the three cases, typically a Newtonian adhesive fails cohesively for large $\varepsilon_{\mathrm{f}}$, whereas an elastic one fails at very small $\varepsilon_{\mathrm{f}}$, smaller than $\varepsilon_{0}$. For a viscoelastic adhesive, $\varepsilon_{\mathrm{f}}$ may depend on $\mathrm{V}$ and lie between the values in the two previous cases. Therefore, the following forces $F(\varepsilon)$ are obtained, corresponding to the Newtonian, viscoelastic strain hardening, and elastic adhesive (Figure 5). 


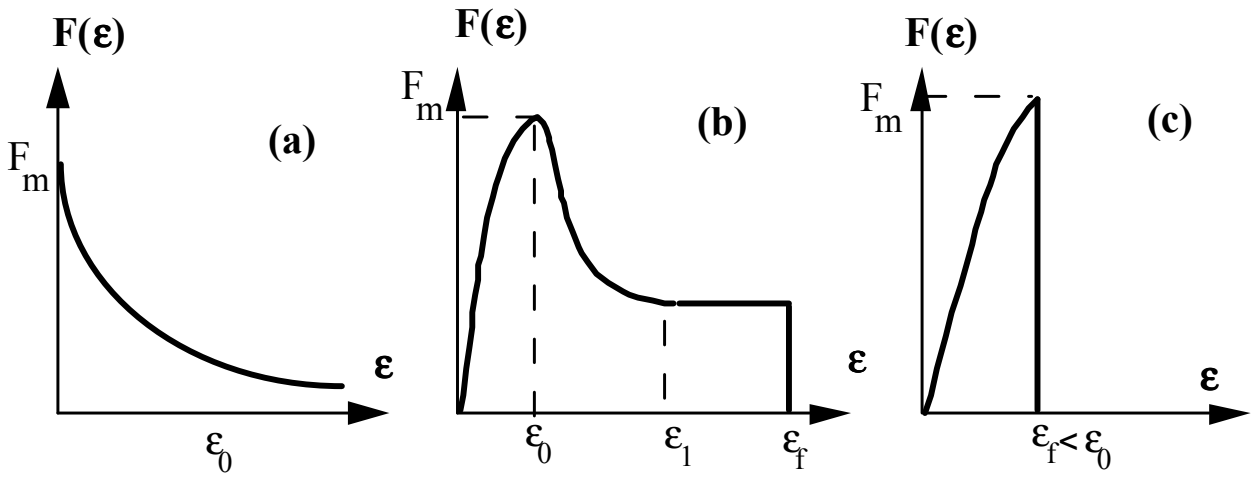

Figure 5. Qualitative predictions of the model (a) Newtonian adhesive (b) viscoelastic strain hardening adhesive $\left(\mathrm{V}=\mathrm{V}_{0}\right)(\mathrm{c})$ elastic adhesive

The Newtonian adhesive "instantaneously" reaches a level of force $F_{m}\left(F_{m}\right.$ is the maximum level of force in the three cases) and then the force decreases as a function of strain. The viscoelastic strain hardening adhesive shows a rapid (elastic) response and the force goes through a maximum then decreases until it reaches a plateau for the case $\mathrm{V}=\mathrm{V}_{0}$. This case is considered because issues have been raised regarding the existence of a so-called plateau [4]. Our model definitely shows that this plateau is due to the strain-hardening effect and can be explained only when considering the proper constitutive equation. In the other cases $\left(V \neq V_{0}\right), F$ can still either decrease $\left(V<V_{0}\right)$, or increase slightly again $\left(\mathrm{V}>\mathrm{V}_{0}\right)$ after the plateau. These behaviors have also been observed in various works from the literature [1-2]. Finally, the elastic adhesive shows an increasing force until it is released from the substrate or probe : crack propagation occurs in such a case [13].

The maximum levels of forces in the three cases are respectively (a) $F_{m}=\sigma_{0} A_{0}$ $F_{m}=E \varepsilon_{0} A_{0} \exp \left(-\varepsilon_{0}\right)(c) F_{m}=A_{0} E \varepsilon_{f} \exp \left(-\varepsilon_{\mathrm{f}}\right)$. They are proportional to the modulus E, in agreement with data from Lakrout et al. [2]. It is not surprising to find this result because the instantaneous response of the adhesive (at small times) is related to the elastic modulus E. 
We conclude that our model retains all the basic features found in the literature, and therefore is a good candidate for predicting tack, especially when elongational mechanisms exist.

\section{Use of a rheological model}

In order to compare this model with actual tack force data $[1,2,13,19]$, we consider Lodge's model, which is well adapted for predicting stresses in viscoelastic materials, in particular when large deformations are involved (i.e. elongation tests). This model has also been shown to predict shear and elongational data quite well for polymeric adhesives [7-8], in particular it can predict strain hardening, which is not the case for all rheological models. Another study can be found [20] where it is shown that the Giesekus model is also a good candidate to predict peel forces, especially because it predicts the transient elongational properties very well in their study. Again, the presence of elongation is clearly emphasized when looking at debonding. Nevertheless, no rheological model has been tested yet in order to predict tack. The constitutive equation for Lodge's model is given by the stress-strain integral function:

$$
\boldsymbol{\Sigma}(\mathrm{t})=\int_{-\infty}^{t} g(t-u) \mathbf{C}_{\mathrm{t}}^{-1}(u) \mathrm{du}
$$

where $\Sigma(t), \mathbf{C}_{\mathrm{t}}^{-1}(\mathrm{t})$ are respectively the Cauchy stress tensor and the inverse of the right Cauchy-Green strain tensor, $\mathrm{g}(\mathrm{t})$ is the relaxation function, and will be simply taken as

$$
g(t)=G \exp (-t / \lambda)
$$

$\mathrm{G}$ is a characteristic modulus and $\lambda$ is the relaxation time. In fact, this single mode relaxation function corresponds to the upper convected Maxwell (UCM) model. Other computations using Wagner or Papanastasiou models [21] have been carried out and show similar trends; they will not be presented here. 
Following the analysis in $\S 4$, the engineering stress $\sigma_{\mathrm{e}}(\mathrm{t})=\mathrm{F}(\mathrm{t}) / \mathrm{A}_{0}$ can be computed using numerical integration with various parameters. The following formula gives the engineering stress, as predicted by (17), in the case of the UCM model, in a constant velocity experiment:

$\sigma_{e}(t)=\frac{e}{e+V t}\left[G e^{-t / \lambda}\left\{\left(\frac{e+V t}{e}\right)^{2}-\left(\frac{e}{e+V t}\right)\right\}+\int_{0}^{t} G e^{-(t-u) / \lambda}\left\{\left(\frac{e+V t}{e+V u}\right)^{2}-\left(\frac{e+V u}{e+V t}\right)\right\} d u\right]$

The values used here are $\mathrm{G}=1 \mathrm{MPa}, \lambda=100 \mathrm{~s}$, and the adhesive thickness is $\mathrm{e}=100 \mu \mathrm{m}$, which are common values taken from other studies $[1,2,13,19]$. The velocity $\mathrm{V}$ is chosen from $0.001 \mu \mathrm{m} / \mathrm{s}$ to $100 \mu \mathrm{m} / \mathrm{s}$, which gives an overall picture. The engineering stress is shown versus time (Figure 6), at different tack velocities, in order to make comparisons with other works. At high velocities (above $0.5 \mu \mathrm{m} / \mathrm{s}$ ), the engineering stress increases very fast and then drops down quite rapidly, in agreement with our model. At intermediate velocities $(0.01$ to $0.1 \mu \mathrm{m} / \mathrm{s})$, the stress increases, reaches a maximum, and then slowly drops down and reaches a plateau, as predicted also. At small velocities, stresses are found to be much smaller, showing reduced tackiness. A typical velocity $\mathrm{V}_{0}$ may be $\mathrm{V}_{0}=0.05 \mu \mathrm{m} / \mathrm{s}$, as defined in $\S 3$, E the elastic modulus $(=3 \mathrm{G})$ is of the order of a few MPa. $\varepsilon_{0}$ and $\varepsilon_{1}$, deduced from the stress or force curve, are respectively of the order 1.5 and 3 , in the intermediate velocity range.

Finally the data shown is quite relevant to similar curves for real adhesives $[1,2,13,19]$, in particular the important case of the viscoelastic adhesive, i.e. the intermediate velocity range $(0.01$ to $0.05 \mu \mathrm{m} / \mathrm{s})$. Here a maximum stress is found to be in the range (0.1 MPa-1 MPa) in agreement with previous data. Concerning the stress hardening effect predicted in the model when $\mathrm{V}>\mathrm{V}_{0}$, it is attributed to the actual deformation of the filaments which actually undergoes higher elongation rates, due to filament necking. These rates need to be corrected with an effective elongation rate which can be in fact 
sometimes six times higher [22] in constant elongational rate experiments; such rates have also been found to be higher in constant force experiments [23]. In the case considered here (constant velocity), it is also very likely that the real elongational rate within filaments is higher, which should give rise to a force increase [24-25] in the final part of the curves in Figure 6.

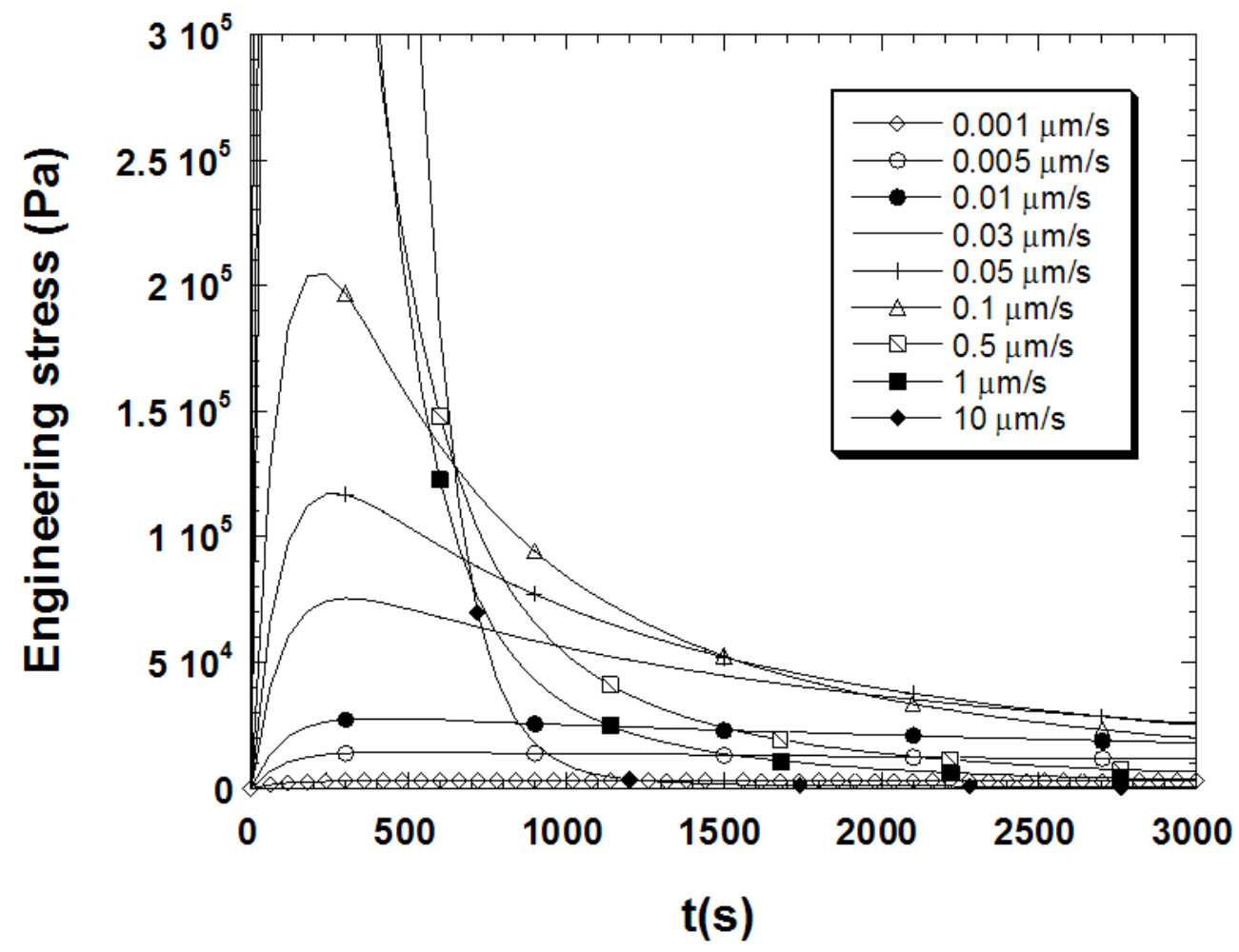

Figure 6. Quantitative predictions using an upper convected Maxwell model: engineering stress vs. time. $\mathrm{G}=1 \mathrm{MPa}, \lambda=100 \mathrm{~s}, \mathrm{~V}$ ranging from $0.001 \mu \mathrm{m} / \mathrm{s}$ to $100 \mu \mathrm{m} / \mathrm{s}$

Data concerning a more complete relaxational spectrum can be easily included in this model (Lodge), but this is not attempted here, since no comparison can be actually given because of the lack of simultaneous measurements of elongation, shear and tack data. Relaxational spectra are important and correspond to the physicochemical properties of the polymeric system (molecular weight $\mathrm{Mw}$, glassy temperature $\mathrm{Tg}$, 
plateau modulus $\mathrm{G}_{\mathrm{N}}{ }^{0}$, etc.), so this model may therefore be a good candidate for testing other experimental results more accurately [1].

\section{Discussion}

\section{Energy}

The energy dissipated in the adhesive is needed to predict the optimal adhesive. One clearly sees that an adhesive in case (b) will give rise to a larger energy. Let us compute $\mathrm{E}_{\mathrm{t}}$, the energy per unit volume, which is the area under the $\sigma-\varepsilon$ curve :

$$
\mathrm{E}_{\mathrm{t}}=\int_{0}^{\varepsilon_{\mathrm{f}}} \sigma(\varepsilon) \mathrm{d} \varepsilon
$$

A simple calculation can show that:

$$
\mathrm{E}_{\mathrm{t}}=\frac{1}{2} \mathrm{E} \varepsilon_{0}^{2}+\sigma_{0}\left(1-\exp \left(\varepsilon_{0}-\varepsilon_{1}\right)\right)+\sigma_{0} \exp \left(\varepsilon_{0}-\varepsilon_{1}\right) \mathrm{V}_{0} / \mathrm{V}\left\{\exp \left[\mathrm{V} / \mathrm{V}_{0}\left(\varepsilon_{\mathrm{f}}-\varepsilon_{1}\right)\right]-1\right\}
$$

This formula contains three terms, one associated with the elastic deformation, the second with the Newtonian response and the last one has the strain hardening effect. When trying to maximize this form, we come up with the final optimal parameters:

1. maximum $\mathrm{E}$ and $\varepsilon_{0}$

2. minimum $\varepsilon_{1}-\varepsilon_{0}$

3. maximum $\varepsilon_{\mathrm{f}}-\varepsilon_{1}$

4. minimum $\mathrm{V}_{0}$

Another way to look at this result is to understand it in terms of the area under the curve in Figure 4d. The height of the curve needs to be maximum so $\mathrm{E}$ and $\varepsilon_{0}$ need to be maximized. Then if $\varepsilon_{1}-\varepsilon_{0}$ is too large, the stress goes to zero too fast so $\varepsilon_{1}-\varepsilon_{0}$ needs to be minimized. Finally, the more strain hardening we have the larger the area, so $\mathrm{V}_{0}$ needs to be small. Indeed the exponential term in (23) will become quite large and will increase tackiness drastically. $\varepsilon_{\mathrm{f}}-\varepsilon_{1}$ needs to be large, of course, because of the increased resistance to motion through stretching. These conditions define a good sticky adhesive.

Nevertheless, we may put a limit to the first condition, because the Dahlquist criterion [26] postulates that $\mathrm{E}$ (or $\mathrm{G}^{\prime}(1 \mathrm{~Hz}$ ), when the glassy temperature $\mathrm{Tg}$ is adjusted) should not be too large. Of course one knows that a very high modulus $\mathrm{E}$ 
means elastic behavior and reduces tackiness because of the absence of good wetting properties. In fact wetting is not included in our model; therefore it is not surprising to have this discrepancy. The combination of both rheological and surface properties is indeed required, and will appear in the final strain.

\section{Final strain $\varepsilon_{\mathbf{f}}$}

The final strain $\varepsilon_{\mathrm{f}}$ can be understood in different ways, according to whether failure is cohesive or adhesive:

- Cohesive failure: the adhesive breaks in the bulk and common criteria have been found for $\varepsilon_{f}$, which is the failure strain. Although the data from Connelly [27] seems to show that $\varepsilon_{\mathrm{f}}$ is almost a constant, it seems that the criterion for the Hencky strain at failure is velocity dependent as shown theoretically [28]. In fact, for such materials, which are rather rubbery, the Considère criterion can be used, as mentioned by McKinley and Hassager [29]. Thus the maximum Hencky strain at failure is strongly dependent on the velocity $\mathrm{V}$, but may show a constant regime at rapid stretching rates. This regime depends on the degree of branching, but to our knowledge no such tack experiments are yet reported which can account for these features. In any case, one should note [29] that increasing the velocity should lead to a higher $\varepsilon_{f}$, whereas above a certain velocity, $\varepsilon_{\mathrm{f}}$ decreases again to a constant level.

- Adhesive failure: in this case, it can be proposed that $\varepsilon_{\mathrm{f}}$ contains the adhesion energy, because filaments may peel off from the substrate (or probe) and they will only do so if the stored energy in the filament is high enough to overcome the adhesion energy. A theory has been proposed previously [15], which holds for an adhesive with an elongational viscosity. A dimensionless number $\Lambda$ (defined in $\S 2.1$ ), representing the effect of this viscosity as compared to the Dupré adhesion energy was used to decide whether failure is possible at the substrate-adhesive interface. This could possibly help for the determination of the final strain $\varepsilon_{\mathrm{f}}$ to be used in (22). Another relevant theory, the so-called «trumpet» regime, for weakly cross-linked adhesives [30] could also be used to predict the final strain $\varepsilon_{f}$, depending on the velocity $\mathrm{V}$. 
In most situations where fibrils are present, a single strain $\varepsilon_{\mathrm{f}}$ is difficult to accept because each fibril is usually different and fibrils are fed non uniformly at their edges by the remaining adhesive. Therefore, one may use a failure strain distribution (gaussian for example). It is not the purpose of this work to go into details here, but it can be guessed that the global force will fall to zero slowly, rather than instantaneously, like in Figures 5b-c. This is what is usually observed in the literature.

The elastic adhesive case is an interesting one, because $\varepsilon_{\mathrm{f}}$ is very small, this is due to the fact that the adhesive remains in one part (no fibrils) and that the high levels of shear contributes to its failure. It is common to develop a crack at the edges [13], running to the middle, in such a way that the force really drops down rather fast, as observed experimentally.

In the Newtonian case, the deformations can be quite large; the filament crosssection can be very small, and the force decreases to zero. No fibrillation is obtained, and usually a long thin filament can be observed - as the force level goes to zero - until it breaks in the middle due to necking [22].

\section{Validity of the model - Improvements}

As mentioned previously, this model is based on a series of hypotheses, which are listed in $\S 4$. These hypotheses are quite reasonable, as far as one is only interested in the force component due to local stretch. Recently, it was shown that stretching does not affect adhesion at a microscopic scale, but that it changes adhesion at a macroscopic scale [31]. In this study, the early aspects of the tack process (i.e. shear motion) have been omitted, but as explained at the end of $\S 2$, may also be accounted for, by considering the shearing flow on a non-newtonian adhesive.

Cavitation effects can of course also be present, due to the high tensions, and to the presence of microvoids present at the wall [2,4], or to surface roughness [5]. They can play a role, but not so important because it is always harder to deform a rubber than an air cavity. Nevertheless it is reasonable to mention that cavities, when present, 
change the adhesive free surface, and therefore affect the deformations within this adhesive. Therefore, accurate measurements of the true contact area are needed $[2,32]$. In the end, the high stresses within the adhesive or rubber lead to high regimes of forces, as observed similarly in peeling experiments [7-10]. In this sense, we believe that this model is realistic.

One other real point of concern is the importance of the surface properties (in particular the Dupré work of adhesion) which are commonly neglected in tack experiments. The influence of the surface energy is far from being understood. Surface energy is difficult to measure, and there is a lack of such data particularly with tack experiments. It is essential to bring it into play, as seen above, because it really controls the peeling of the fibrils, especially at the end of the tack experiment. This is in fact similar to Saint-Venant's problem [33], where the base fixation is ignored, until it breaks, in other words when one reaches $\varepsilon_{\mathrm{f}}$. The careful experimentalist needs to take all these factors into account, in order to improve the knowledge of tack.

\section{Conclusion}

This work deals with tack while trying to bring a new insight, based on the influence of the elongational properties of commonly used adhesives. A model has been proposed, which predicts the usual features of the nonlinear tack force found experimentally in the literature. Limiting cases of elastic and Newtonian adhesives have also been considered.

The main result concerns the maximum tackiness and provides the shape of an ideal adhesive stress-strain curve, which can be obtained rather easily on an elongational rheometer. The ideal adhesive must undergo strain hardening, while other parameters of the model need to be adjusted.

Finally, it is concluded that an optimal model should account for surface energies, roughness, cavitation, as well as the proper rheological properties. 


\section{REFERENCES}

[1] Zosel, A. Int J Adhesion and Adhesives 1989, 18, 265-271.

[2] Lakrout, H.; Sergot, P.; Creton, C. J Adhesion 1999, 69, 307-359.

[3] Crosby, A.J. ; Shull, K.R., Adhesives Age 1999, 42, 28-33.

[4] Gay, C.; Leibler, L. Phys Rev Letters 1999, 82, 936-939.

[5] Creton, C.; Leibler, L. J Polym Sci Polym Phys Ed 1996, 34, 545-554.

[6] Hui, C.Y.; Lin, Y. Y.; Baney, J. M. J Polym Sci Polym Phys Ed 2000, 38, $1485-$ 1495.

[7] Verdier, C.; Piau, J. M.; Benyahia, L. C R Acad Sci Paris IIb 1996, 323, 739-746.

[8] Piau, J. M.; Verdier, C.; Benyahia, L. Rheol Acta 1997, 36, 449-461.

[9] Benyahia, L.; Verdier, C.; Piau, J. M. J Adhesion 1997, 62, 45-73.

[10] Kaelble, D. H. Trans Soc Rheol 1965, 9, 135-163.

[11] Gay, C. ; Leibler, L. ; Physics Today 1999, 52, 48-52.

[12] Ondarçuhu, T. J Phys II France 1997, 7, 1893-1916.

[13] Crosby, A. J. ; Shull, K. R. ; Lakrout, H. ; Creton, C. J Appl Phys 2000, 88, 29562966.

[14] Gent, A. N.; Kim, H. J. Rubber Chem Technol 1990, 63, 613-623.

[15] Good, R. J.; Gupta, R. K. J Adhesion 1988, 26, 13-36.

[16] Gent, A. N.; Schultz, J. J Adhesion 1972, 3, 281-294.

[17] Chikina, I. ; Gay, C. Phys Rev Letters 2000, 85, 4546-4549.

[18] Connelly, R. W.; Garfield, L. J.; Pearson, G. H. J Rheol 1979, 23, 651-662.

[19] Crosby, A. J. ; Shull, K.R. J Polym Sci B :Polym Phys 1999, 37, 3455-3472.

[20] Christensen, S.F. ; McKinley, G.H. Int J Adhesion Adhesives 1998, 18, 333-343.

[21] Larson, R. G. In «Rheology, Principles, Measurements and applications », Macosko, C.W., 1994, Wiley-VCH, Chapter 4, pp 158-165. 
[22] Yao, M. ; McKinley, G. H. ; Debbaut, B. J Non-Newtonian Fluid Mech 1998, 79, 469-501.

[23] Mestadi, A. ; Normandin, M. ; Clermont, J.R. ; Attané, P. Rheol Acta 1996, 35, 337-346.

[24] Spiegelberg, S. H. ; Ables, D.C. ; McKinley, G.H. J Non-Newtonian Fluid Mech $1996,64,229-267$.

[25] McKinley, G.H., Proc XIIIth Int. Congress on Rheology (vol I), Cambridge, UK, Aug. 2000, pp 15-22.

[26] Dahlquist, C.A.; in: Treatise on adhesion and adhesives: Patrick, R.L. (Ed.)., NewYork: Marcel Dekker (1969).

[27] Connelly, R. W.; Parsons, W. F.; Pearson, G. H. J Rheol 1981, 25, 315-328.

[28] Malkin, A.Y. ; Petrie, C.S.J. J Rheol 1997, 41, 1-25.

[29] McKinley, G.H. ; Hassager, O. J Rheol 1999, 43, 1195-1212.

[30] de Gennes, P. G. C R Acad Sci IIb 1988, 307, 1949-1953.

[31] Gay, C. Int J Adhesion Adhesives 2000, 20, 387-393.

[32] Tordjeman, P.; Papon, E.; Villenave, J.J. J Polym Sci B :Polym Phys 2000, 38, 1201-1208.

[33] Lakes, R. S. In « Viscoelastic Solids »; CRC Press : Boca Raton, 1999 ; Chapter 5, pp 151-152. 